\title{
Usefulness of Contralateral Indirect Decompression through Minimally Invasive Unilateral Transforaminal Lumbar Interbody Fusion
}

\author{
Sang-Hyuk Min, Jae-Sung Yoo, Jun-Yeul Lee \\ Department of Othopedic Surgery, Dankook University College of Medicine, Cheonan, Korea
}

\begin{abstract}
Study Design: Retrospective study.
Purpose: This study aims to investigate the clinical and radiological results of contralateral indirect decompression through minimally invasive unilateral transforaminal lumbar interbody fusion (MI-TLIF).

Overview of Literature: Several studies have proposed that blood loss and operation time could be reduced through a unilateral approach, although many surgeons have forecast that satisfactory foraminal decompression is difficult to achieve through a unilateral approach.

Methods: The study included 30 subjects who had undergone single-level MI-TLIF. Visual analogue scale (VAS) and Oswestry disability index (ODI) were analyzed for clinical assessment. Disc height, segmental lordosis, and lumbar lordosis angle were examined for radiological assessment. The degree of contralateral indirect decompression was evaluated through a comparative analysis, with a magnetic resonance imaging (MRI) performed preoperatively and at one year postoperatively.

Results: Intraoperative blood loss volume was $308.75 \mathrm{~mL}$ in the unilateral approach group (UAP), and $575.00 \mathrm{~mL}$ in the bilateral approach group (BAP), showing a statistically significant difference. Operation time was 139.50 minutes in the UAP group, and 189.00 minutes in the BAP group, exhibiting a statistically significant difference $(p<0.05)$. On the other hand, no significant difference was found in VAS, ODI, disc height, lordosis angles and the degree of nerve decompression in the vertebral foramen, using MRI, between the two groups $(p>0.05)$

Conclusions: Satisfactory results were acquired with MI-TLIF conducted through the unilateral approach of contralateral indirect decompression, in alignment with the bilateral approach. Therefore, contralateral indirect decompression is thought to be a useful procedure in reducing the operation time and volume of blood loss.
\end{abstract}

Keywords: Spinal fusion; Surgical procedures, Minimally invasive; Unilateral approach

\section{Introduction}

Several studies have reported foraminal decompression and interbody fusion performed using minimally invasive transforaminal lumbar interbody fusion (MI-TLIF) to improve some demerits of interbody fusion by the conventional posterior approach, such as reducing soft tissue injury and muscle atrophy. Although MI-TLIF minimizes complications such as postoperative back pain and muscle atrophy, and decreases the volume of blood loss,

Received Jun 13, 2013; Revised Sep 1, 2013; Accepted Sep 1, 2013

Corresponding author: Sang-Hyuk Min

Department of Orthopaedic Surgery, Dankook University College of Medicine,

201 Manghyang-ro, Dongnam-gu, Cheonan 330-180, Korea

Tel: +82-41-550-3950, Fax: +82-41-556-3238, E-mail: idbabe@hanmail.net 
Table 1. Patient's data

\begin{tabular}{lccc} 
& Overall & Unilateral approach $(\mathrm{n}=20)$ & Bilateral approach $(\mathrm{n}=10)$ \\
Age $(\mathrm{yr})$ & $56.10(30-75)$ & $56.60(40-75)$ & $55.10(30-70)$ \\
Male:female & $11: 19$ & $5: 15$ & $6: 4$ \\
Follow-up (mo) & $24.53(12-52)$ & $25.24(12-48)$ & $23.46(12-52)$ \\
Body mass index & $22.81(17.72-29.73)$ & $23.92(17.72-29.73)$ & $24.32(20.57-29.35)$ \\
Bone mineral density (hip) & $-0.76(-3.70-1.20)$ & $-0.66(-2.61-1.00)$ & $-0.95(-3.70-1.20)$ \\
\hline
\end{tabular}

it requires a longer operation time than the conventional posterior approach [1-7].

To resolve this limitation, several studies have recently proposed that the blood loss and the operation time could be reduced through a unilateral approach by making a single paramedian skin incision [8,9]. A large number of surgeons have forecast that satisfactory foraminal decompression would be difficult to achieve through a unilateral approach using contralateral indirect decompression.

The purpose of this study was to investigate the clinical and radiological results of the unilateral approach of contralateral indirect decompression, by analyzing and comparing it with the bilateral approach.

\section{Materials and Methods}

\section{Subjects}

This study included 30 subjects (unilateral approach, 20 patients; bilateral approach, 10 patients) who had been followed up for at least a year after undergoing singlelevel MI-TLIF due to the chief complaint of radiating pain in both legs, in our hospital from March 2006 to June 2011. The average follow-up period was 18.6 months (range, 12-48 months) (Table 1). The surgical indications were low back pain and pain radiating down to the lower extremity (leg pain) associated with a single-level lumbar or lumbosacral segmental instability of low grade (grade I/II) spondylolytic or degenerative spondylolisthesis and degenerative segmental instability combined with spinal stenosis and/or disc herniation. The inclusion criteria of segmental instability was $\geq 4 \mathrm{~mm}$ of translation or $\geq 10$ $\mathrm{mm}$ of angular motion on preoperative flexion and extension radiographs. However, patients with symptomatic radiological instability in $>1$ segment, a previous history of spondylodiscitis or a pathological condition of the lumbar spine (e.g., trauma or tumor) were excluded. Patients with high-grade (grade III/IV) spondylolisthesis were also excluded because of the unavailability of reduction pedicle screws and in situ reduction devices.

The unilateral and bilateral approaches were chosen according to the period without the other selection criteria. The bilateral approach was performed in the first-half of the period and the unilateral approach was performed in the latter-half of the period. The direction of fusion in the unilateral approach was decided according to the site with severe preoperative clinical symptoms. In the case of similar clinical symptoms in more than one site, the site with severe stenosis according to the radiological examination was chosen.

\section{Surgical methods}

In the bilateral approach group, the spine was bilaterally approached through two $2.5 \mathrm{~cm}$-long incisions in the area about $2.5 \mathrm{~cm}$ distant from the midline of the back, and the multifidus muscles and longissimus dorsi were stripped off. After making an approach between those muscles, a tubular retractor was hung, which reached the lamina of the vertebral arch and the facet joint. Under microscopic visualization, the inferior articular facet and the upper half of the superior articular facet were removed and then lumbar discectomy was performed after exposing the neuromuscular and spinal canal dura mater by removing the ligamentum flavum. Subsequently, the interbody spaces were consecutively extended using a shaver, and curettage of the endplate was performed to prepare the fused superior and inferior lumbar endplates. After performing the same procedures on the other side, a cage filled with bone fragments obtained from the decompression was inserted into the disc space. After performing identical procedures on the contralateral side, percutaneous pedicle screw fixation was performed. 
In the unilateral approach group (UAP), the spine was unilaterally approached through a $2.5 \mathrm{~cm}$-long incision in the area about $2.5 \mathrm{~cm}$ distant from the midline of the back, and the multifidus muscles and longissimus dorsi were stripped off. By making an approach between those muscles, a tubular retractor was hung reaching the lamina of the vertebral arch and the facet joint. Under microscopic visualization, the inferior articular facet and the upper half of the superior articular facet were removed and then lumbar discectomy was performed after exposing the neuromuscular and spinal canal dura mater by removing the ligamentum flavum. Subsequently, the interbody spaces were consecutively extended using a shaver, and curettage of the endplate was performed to prepare the fused superior and inferior lumbar endplates. Patients with spinal stenosis were positioned to the opposite side of the surgeon, and sublaminar decompression was unilaterally performed using a high-speed drill under microscopic visualization. After confirming sufficient neural decompression, the disc spaces were filled with the bone fragments obtained from the laminectomy using allograft bone obtained from the posterior-superior iliac spine or with artificial bone synthesized from hydroxyapatite. Consequently, a cage made of autogenous bone was then inserted into the disc space, and percutaneous pedicle screw fixation was performed (Fig. 1).

\section{Analysis methods}

Patients who underwent single-level MI-TLIF were classified into 20 subjects in the UAP and 10 subjects were classified in the bilateral approach, according to the surgical technique used. The subjects were compared by dividing them into two groups of ten. The subjects were analyzed based on sex, age, medical history (blood pressure, diabetes, and endocrine disease), height, weight, body mass index (BMI), and bone mineral density (BMD).

For the clinical and radiological evaluation, postoperative improvement was compared and analyzed according to the preoperative state, the state at two postoperative weeks, and the state at the last follow-up examination. Intraoperative bleeding and drainage volumes, operation time, visual analogue scale (VAS) score, and Oswestry disability index (ODI) were analyzed for the clinical assessment.

For the radiological assessment, radiological findings (disc height, sagittal segmental lordosis, and lumbar lordosis) preoperatively, at two postoperative weeks, and at the last follow-up visit were evaluated using simple radiographs of the Picture Archiving Communication System. The degree of nerve compression in the vertebral foramen was evaluated with an MRI.

A straight line connecting the superior and inferior
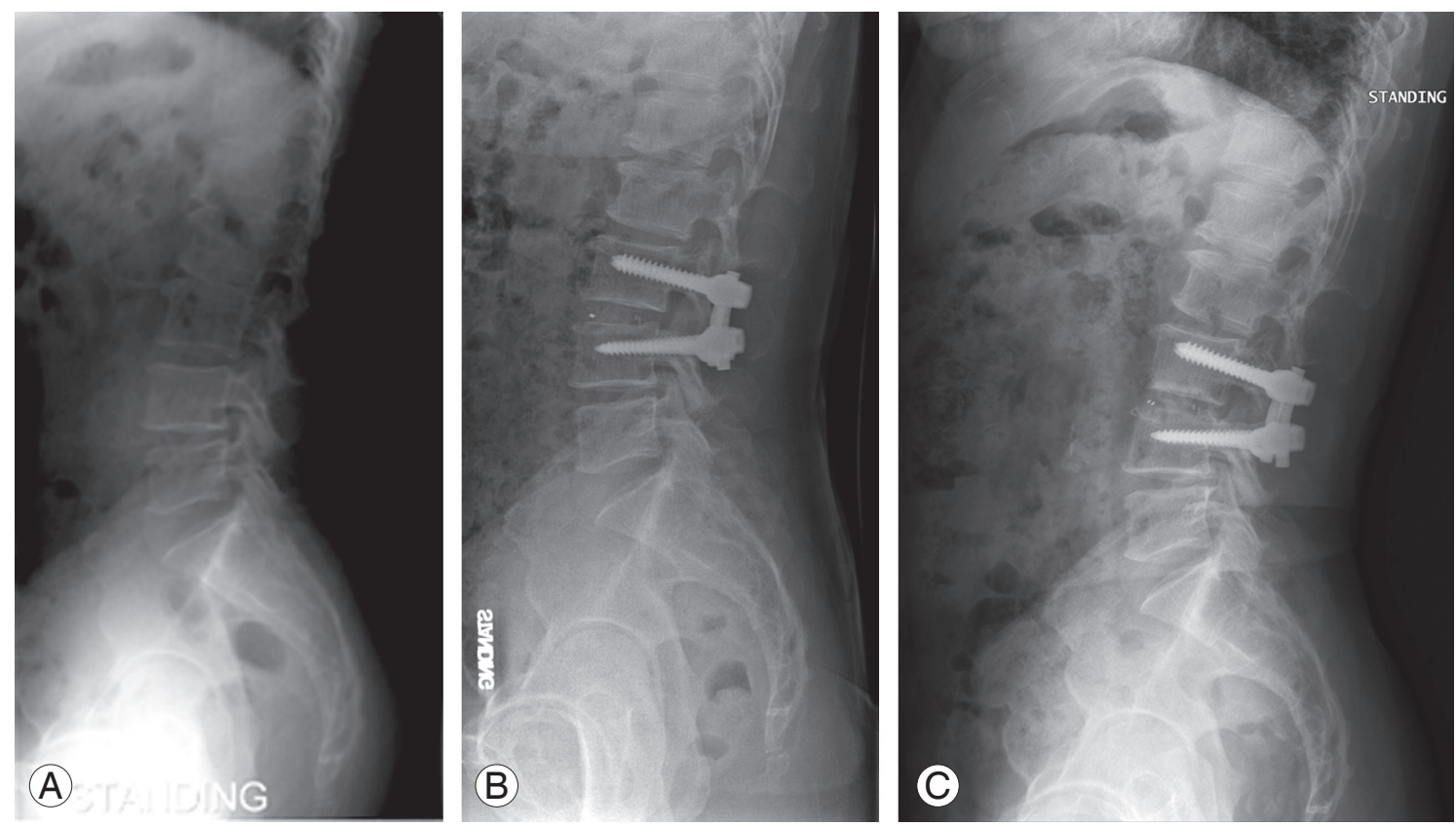

Fig. 1. Unilateral minimally invasive transforaminal lumbar interbody fusion procedure. (A) Preoperative radiography. (B) Postoperative radiography. (C) Last follow-up radiography. 
epiphyseal plates was drawn at the segments on which the fusion was performed, and defined as the length between the perpendiculars connecting the centers of the epiphyseal plates. The segmental lordosis at L5-S1 was defined as the angle subtended by the superior endplate line of L5 and the superior endplate line of S1. The degree of lumbar lordosis was defined as the angle subtended by the superior endplate line of L1 and the superior endplate line of S1 (Fig. 2). The average value of the two measurements made by an orthopedic surgeon who specialized in the spinal column and a radiologist who specialized in the musculoskeletal system was used. To evaluate the degrees of postoperative radiological correction and correction loss, differences in the radiological measurements were compared and analyzed according to the measurements preoperatively, at two postoperative weeks, and at the last follow-up.

The degree of nerve compression in the vertebral foramen was assessed based on the MRI images taken preoperatively and at one postoperative year, and classified into the grades of $0,1,2$, and 3 according to the Kunogi and Hasue classification (Fig. 3) [10]. The classification was determined by an orthopedic surgeon who specialized in the spinal column and a radiologist who specialized in the musculoskeletal system. The grade was evaluated only when the measurements of the two specialists coincided. A lower grade was given in the case where the measurements of the two specialists did not match. Furthermore, differences in the degree of foramen compression that was assessed based on the Kunogi and Hasue [10] clas-
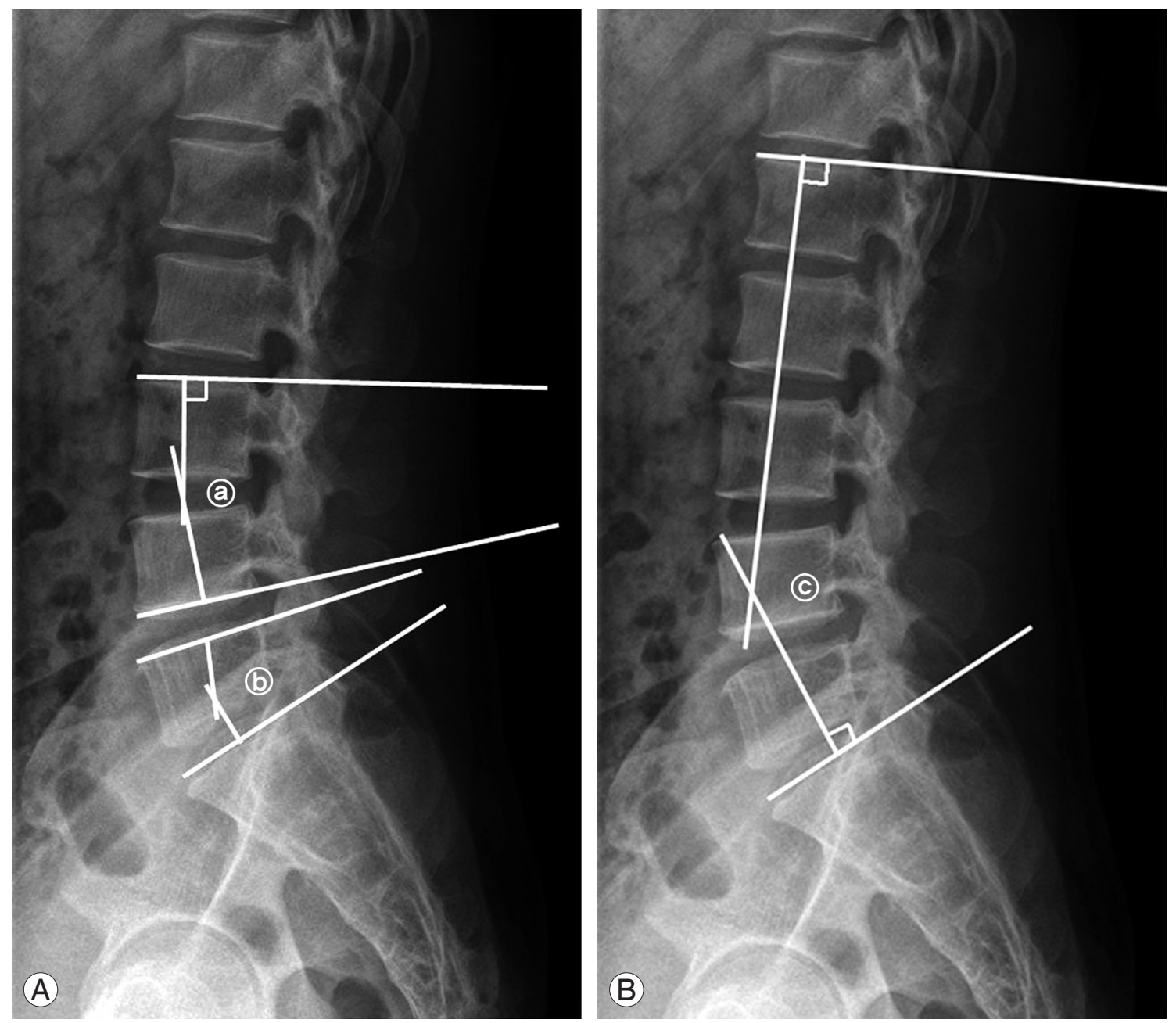

Fig. 2. Cobb's angle for segmental lordosis and whole lumbar lordosis. (A) The segmental lordosis at L3-4 (a) was defined as the angle subtended by the superior endplate line of $\mathrm{L} 3$ and the inferior endplate line of $L 4$. The segmental lordosis at L5-S1 (b) was defined as the angle subtended by the superior endplate line of $L 5$ and the superior endplate line of $S 1$. (B) Whole lumbar lordosis (C) was defined as the angle subtended by the superior endplate line of L1 and the superior endplate line of S1. 
Table 2. Clinical results

\begin{tabular}{lcc} 
& Unilateral approach $(\mathrm{n}=20)$ & Bilateral approach $(\mathrm{n}=10)$ \\
\hline Average of surgical time & $139.50 \pm 5.26$ & $189.00 \pm 7.29$ \\
\hline Average of intraoperative blood loss & $308.75 \pm 30.52$ & $575.00 \pm 61.57$ \\
\hline Average of postoperative drainage & $113.45 \pm 15.78$ & $221.15 \pm 48.20$ \\
Visual analogue scale (leg pain) & $7.50 \pm 0.51$ & $6.50 \pm 0.79$ \\
\hline Preoperation & $2.25 \pm 0.56$ & $1.30 \pm 0.30$ \\
\hline Postoperation & $0.40 \pm 0.15$ & $0.90 \pm 0.28$ \\
\hline Last follow-up & & $23.40 \pm 3.40$ \\
\hline Oswestry disability index & $23.60 \pm 2.34$ & $12.30 \pm 2.948$ \\
\hline Preoperation & $9.90 \pm 8.17$ & $8.50 \pm 2.69$ \\
\hline Postoperation & $7.35 \pm 1.47$ & \\
\hline Last follow-up & & \\
\hline
\end{tabular}

sification were compared and analyzed between the preoperative and one postoperative year states for the evaluation of the degree of postoperative nerve compression.

\section{Statistical analysis}

Student's $t$-test and the chi-square test were performed for statistical analysis using Window SPSS ver. 19.0 (IBM Co., Armonk, NY, USA). Age, sex, medical history, BMI, and BMD were modified. $p$-values less than 0.05 were considered statistically significant.

\section{Results}

\section{Clinical results and evaluation}

The mean operation time was 139.50 minutes in the UAP, and 189.0 minutes in the bilateral approach group (BAP), exhibiting a statistically significant difference $(p<0.05)$. The average intraoperative blood loss volume was 308.75 $\mathrm{mL}$ in the UAP group and $575.00 \mathrm{~mL}$ in the BAP group, showing a statistically significant difference $(p<0.05)$ (Table 2). Moreover, the average postoperative drainage volume was $113.45 \mathrm{~mL}$ in the UAP group and 221.15
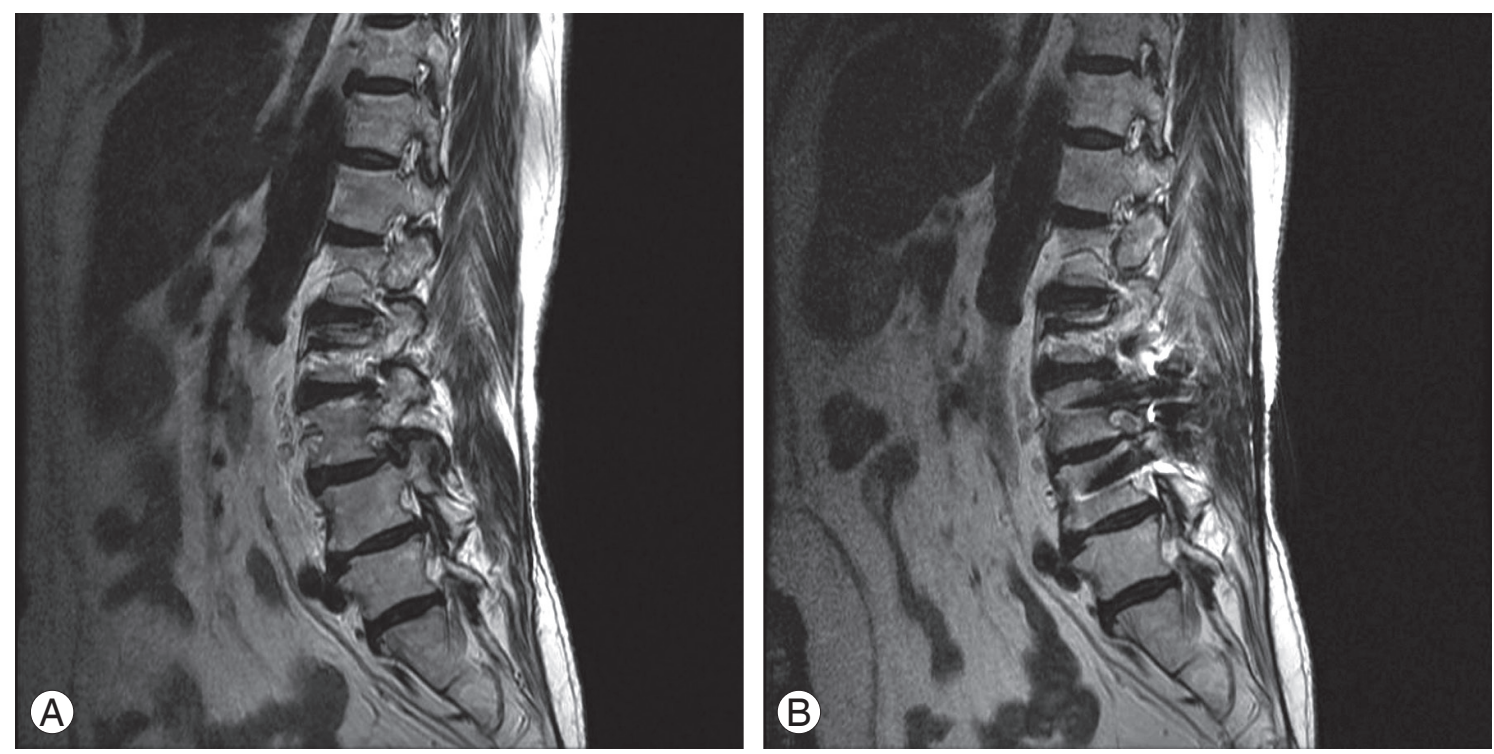

Fig. 3. Unilateral minimally invasive transforaminal lumbar interbody fusion procedure. (A) Preoperative magnetic resonance imaging showed severe contralateral foraminal stenosis. (B) Postoperative radiography showed neural decompression. 
Table 3. Radiologic finding

\begin{tabular}{lcc} 
Characteristic & Unilateral approach $(\mathrm{n}=20)$ & Bilateral approach $(\mathrm{n}=10)$ \\
Disc height $(\mathrm{mm})$ & & $10.05 \pm 0.68$ \\
\hline Preoperation & $9.21 \pm 0.49$ & $12.55 \pm 0.73$ \\
\hline Postoperation & $11.68 \pm 0.66$ & $11.90 \pm 0.96$ \\
\hline Last follow-up & $10.22 \pm 0.50$ & $17.90 \pm 2.30$ \\
\hline Segmental lordotic angle $\left(^{\circ}\right)$ & & $20.70 \pm 2.24$ \\
\hline Preoperation & $15.05 \pm 1.62$ & $18.70 \pm 1.58$ \\
\hline Postoperation & $18.60 \pm 1.37$ & $41.30 \pm 7.62$ \\
\hline Last follow-up & $17.65 \pm 1.10$ & $47.50 \pm 5.37$ \\
\hline Lumbar lordotic angle $\left(^{\circ}\right)$ & & $44.80 \pm 5.87$ \\
\hline Preoperation & $29.85 \pm 3.10$ & $37.25 \pm 2.13$ \\
\hline Postoperation & $35.80 \pm 2.69$ & \\
\hline Last follow-up & & \\
\hline
\end{tabular}

$\mathrm{mL}$ in the BAP group, showing a statistically significant difference $(p<0.05)$.

The VAS scores of the lower extremities significantly improved from an average of 7.50 points preoperatively to an average of 2.25 points at the second postoperative week in the UAP group. The score improved to 0.40 point at the last follow-up. The VAS scores in the BAP group improved from an average of 6.50 points preoperatively to an average of 1.30 points at the second postoperative week, and increased to 0.90 point at the last follow-up. No significant differences were found $(p>0.05)$ (Table 2).

ODI exhibited an increasing trend, from an average of 23.60 points preoperatively to an average of 9.90 points at the second postoperative week in the UAP group. The ODI improved up to 7.35 points at the last follow-up. In the BAP group, the ODI increased from an average of 23.40 points preoperatively to an average of 12.30 points at the second postoperative week, and improved to an average of 10.60 at the last follow-up. No significant differences were shown between the groups $(p>0.05)$ (Table 2).

\section{Radiological results and evaluation}

The average disc heights were $9.21 \mathrm{~mm}$ preoperatively, $11.68 \mathrm{~mm}$ at the second postoperative week, and 10.22 $\mathrm{mm}$ at the last follow-up in the UAP group. The average disc heights were $10.05 \mathrm{~mm}$ preoperatively, $12.55 \mathrm{~mm}$ at the second postoperative week, and $11.90 \mathrm{~mm}$ at the last follow up in the BAP group. The average postoperative degrees of correction were $2.47 \mathrm{~mm}$ in the UAP group and $2.50 \mathrm{~mm}$ in the BAP group. The averages of correction loss were $1.46 \mathrm{~mm}$ in the UAP group and $0.65 \mathrm{~mm}$ in the BAP group, showing no significant difference $(p>0.05)$ (Table 3).

The mean segmental lordosis angles were $15.05^{\circ}$ preoperatively, $18.06^{\circ}$ at the second postoperative week, and $17.65^{\circ}$ at the last follow-up in the UAP group. The mean segmental lordosis angles were $17.90^{\circ}$ preoperatively, $20.70^{\circ}$ at the second postoperative week, and $18.70^{\circ}$ at the last follow-up in the BAP group. The average postoperative angles of correction were $3.01^{\circ}$ in the UAP group and $2.80^{\circ}$ in the BAP group. The average angles of correction loss were $0.41^{\circ}$ in the UAP group and $2.00^{\circ}$ in the BAP group, showing no significant difference $(p>0.05)$ (Table 3).

The mean lumbar lordosis angles were $29.85^{\circ}$ preoperatively, $37.25^{\circ}$ at the second postoperative week, and $35.80^{\circ}$ at the last follow-up in the UAP group. The mean lumbar lordosis angles were $41.30^{\circ}$ preoperatively, $41.30^{\circ}$ at the second postoperative week, and $44.80^{\circ}$ at the last follow up in the BAP group. The average postoperative angles of correction were $7.40^{\circ}$ in the UAP group and $6.20^{\circ}$ in the BAP group. The average angles of correction loss were $1.45^{\circ}$ in the UAP group and $2.70^{\circ}$ in the BAP group, showing no significant difference $(p>0.05)$ (Table 3).

The differences in the degree of nerve compression in the vertebral foramen were compared and analyzed to assess the improvement of grades classified based on the 
Table 4. Foraminal stenosis of contra-lateral vertebral foramen (Kunogi and Hasue classification)

\begin{tabular}{|c|c|c|}
\hline Magnetic resonance imaging & Unilateral approach $(\mathrm{n}=20)$ & Bilateral approach $(n=10)$ \\
\hline \multicolumn{3}{|l|}{ Preoperative } \\
\hline Grade 0 & 0 & 0 \\
\hline Grade 1 & 4 & 2 \\
\hline Grade 2 & 6 & 3 \\
\hline Grade 3 & 10 & 5 \\
\hline \multicolumn{3}{|l|}{ Postoperative } \\
\hline Grade 0 & 7 & 4 \\
\hline Grade 1 & 13 & 5 \\
\hline Grade 2 & 0 & 0 \\
\hline Grade 3 & 0 & 1 \\
\hline
\end{tabular}

Kunogi and Hasue classification using MRI scanning conducted preoperatively and at one postoperative year. Preoperative and postoperative differences in the grade were an average of 1.65 in the UAP group, and an average of 1.50 in the BAP group, exhibiting no significant differences $(p>0.05)$ (Table 4$)$.

\section{Complications}

The degree of interbody fusion was assessed according to the presence of the bony bridge connecting the upper and lower vertebral bodies and the anterior and posterior cages based on a simple X-ray, the absence of radiological dissection between the cage and the vertebral endplate, the absence of destruction or dissection of the pedicle screw system, and motion of less than $2^{\circ}$ in the flexionextension movement image. A case of non-union was observed in UAP and BAP each. No complications such as damage to the dura mater, infection, or neural symptoms occurred.

\section{Discussion}

Intraoperative blood loss volume increases due to a soft tissue injury or excessive traction generated by an extensive approach including the approaches of posterior fusion or posterior lumbar interbody fusion using conventional pedicle screws, and this leads to the occurrence of complications and a delay in postoperative recovery [11-15], On the other hand, in MI-TLIF, there is minimization of injuries to the soft tissues and the surrounding muscle, because the approach is made between the multifidus muscles and longissimus dorsi after separating them, and the intervertebral disc is reached from the lateral vertebral foramen, reducing the chances of excessive traction. The advantages of this surgical procedure are the minimization of muscle or soft tissue injuries that are incurred by the lateral approach, reduction of surgically related muscle damage, and a decrease of the postoperative blood loss [1-6,16-18].

However, Foley et al. [1] suggested that the recognition of surgical landmarks is complicated compared to conventional surgery due to the limited operational space of MI-TLIF and understanding of the vertebral structures and the 3-dimensional neural network. In addition, the acquisition of surgical techniques takes a longer period of time, such as becoming accustomed to using a microscope and the surgical instruments involved. Foley et al. [1] reported that the operation time was an average of 240 minutes for the completion of MI-TLIF and percutaneous pedicle screw fixation in the case of 12 patients.

Various attempts have been made to reduce the volume of bleeding and the operation time. Min and Lee [8] and Min and Hwang [9] reported that the unilateral approach showed a statistically significant decrease in the bleeding volume and operation time compared to the bilateral approach. A large number of surgeons consider that performing contralateral indirect decompression through a unilateral approach is insufficient for acquiring desirable foramen decompression. Inoue et al. [19] highlighted the importance of recovery of the disc height and anatomical realignment of the spinal canal in obtaining a favorable postoperative clinical outcome. Jeon et al. [20] reported that increases in the diameter and the space of the neural 
foramen postoperatively indicated sufficient decompression, leading to good clinical results. According to the results of this study, no significant differences were detected in the recovery of the disc height and segmental lordosis and lumbar lordosis angles between the two groups. Moreover, no significant differences were found in the improvement of nerve compression using the MRI and the VAS scores of the lower extremities between the two groups. The outcomes explain the successful contralateral decompression through interbody extension in the unilateral approach by indirect decompression.

There are some limitations in this study. This study had some selection bias because it was not a prospective randomized study but a retrospective one. Moreover, this was a small-scale retrospective study with a shortterm follow-up period. However, the study is meaningful as it is the first investigation to compare the clinical and radiological results of contralateral decompression of MITLIF through the unilateral approach with those of the bilateral approach, using MRI scans.

\section{Conclusions}

Satisfactory results were obtained in MI-TLIF conducted through the unilateral approach of contralateral indirect decompression, aligning with the bilateral approach. When the limitations of the unilateral approach are resolved and surgeons become accustomed to the surgical techniques, contralateral indirect decompression is thought to be a useful procedure in reducing the operation time and volume of blood loss.

\section{Conflict of Interest}

No potential conflict of interest relevant to this article was reported.

\section{References}

1. Foley KT, Holly LT, Schwender JD. Minimally invasive lumbar fusion. Spine (Phila Pa 1976) 2003;28:S26-35.

2. Peng CW, Yue WM, Poh SY, Yeo W, Tan SB. Clini$\mathrm{cal}$ and radiological outcomes of minimally invasive versus open transforaminal lumbar interbody fusion. Spine (Phila Pa 1976) 2009;34:1385-9.

3. Holly LT, Schwender JD, Rouben DP, Foley KT.
Minimally invasive transforaminal lumbar interbody fusion: indications, technique, and complications. Neurosurg Focus 2006;20:E6.

4. Kim JS, Jung B, Lee SH. Instrumented minimally invasive spinal-transforaminal lumbar interbody fusion (MIS-TLIF); minimum 5-years follow-up with clinical and radiologic outcomes. J Spinal Disord Tech 2012 Sep 28 [Epub]. http://dx.doi.org/10.1097/ BSD.0b013e31827415cd.

5. Foley KT, Gupta SK. Percutaneous pedicle screw fixation of the lumbar spine: preliminary clinical results. J Neurosurg 2002;97:7-12.

6. Foley KT, Lefkowitz MA. Advances in minimally invasive spine surgery. Clin Neurosurg 2002;49:499517.

7. Kim JS, Kang BU, Lee SH, et al. Mini-transforaminal lumbar interbody fusion versus anterior lumbar interbody fusion augmented by percutaneous pedicle screw fixation: a comparison of surgical outcomes in adult low-grade isthmic spondylolisthesis. J Spinal Disord Tech 2009;22:114-21.

8. Min SH, Lee DH. Minimally invasive transforaminal lumbar interbody fusion in patients with low grade spondylolisthesis: comparison of the unilateral and bilateral approaches. J Korean Orthop Assoc 2009;44:429-35.

9. Min SH, Hwang SS. Minimal invasive unilateral transforaminal lumbar interbody fusion by sublaminar decompression: comparison to bilateral approach. J Korean Orthop Assoc 2009;44:76-82.

10. Kunogi J, Hasue M. Diagnosis and operative treatment of intraforaminal and extraforaminal nerve root compression. Spine (Phila Pa 1976) 1991;16:1312-20.

11. Steffee AD, Sitkowski DJ. Posterior lumbar interbody fusion and plates. Clin Orthop Relat Res 1988;227:99102.

12. McLaughlin MR, Haid RW Jr, Rodts GE Jr, Subach BR. Posterior lumbar interbody fusion: indications, techniques, and results. Clin Neurosurg 2000;47:51427.

13. Suk SI, Lee CK, Kim WJ, Lee JH, Cho KJ, Kim HG. Adding posterior lumbar interbody fusion to pedicle screw fixation and posterolateral fusion after decompression in spondylolytic spondylolisthesis. Spine (Phila Pa 1976) 1997;22:210-9.

14. Deyo RA, Cherkin DC, Loeser JD, Bigos SJ, Ciol MA. Morbidity and mortality in association with 
operations on the lumbar spine. The influence of age, diagnosis, and procedure. J Bone Joint Surg Am 1992;74:536-43.

15. Katz JN, Lipson SJ, Brick GW, et al. Clinical correlates of patient satisfaction after laminectomy for degenerative lumbar spinal stenosis. Spine (Phila Pa 1976) $1995 ; 20: 1155-60$.

16. Khoo LT, Palmer S, Laich DT, Fessler RG. Minimally invasive percutaneous posterior lumbar interbody fusion. Neurosurgery 2002;51 (5 Suppl):S166-1.

17. Bagan B, Patel N, Deutsch H, et al. Perioperative complications of minimally invasive surgery (MIS): comparison of MIS and open interbody fusion techniques. Surg Technol Int 2008;17:281-6.
18. Villavicencio AT, Burneikiene S, Roeca CM, Nelson EL, Mason A. Minimally invasive versus open transforaminal lumbar interbody fusion. Surg Neurol Int 2010;1:12.

19. Inoue $\mathrm{S}$, Watanabe $\mathrm{T}$, Hirose $\mathrm{A}$, et al. Anterior discectomy and interbody fusion for lumbar disc herniation. A review of 350 cases. Clin Orthop Relat Res 1984;(183):22-31.

20. Jeon CH, Kim YC, Chang HG, Kim YW, Jung NS, Shin DS. The comparison of changes in the dimensions of the intervertebral disc and neural foramen between anterior lumbar interbody fusion and posterolateral fusion in the lumbar spine. J Korean Soc Spine Surg 2007;14:263-9. 